\title{
22. Zwischenfazit für Standortbestimmung und Ausblick
}

Die letzte Teilfrage „Wird die Stabilisierung nach der Landtagswahl 2016 fortbestehen?" ist keine Entscheidungsfrage, die mit Ja oder Nein beantwortet werden kann. Das Ergebnis der Standortanalyse und des Ausblicks der Arbeit ist, dass die aktuelle Stabilität ein wackliges Konstrukt ist. Denn im letzten Teil V wurde analysiert, ob die Eigenschaftskonstanten, die die aktuelle Stabilität stützen, nach 2016 noch Bestand haben. Das Ergebnis war hinsichtlich der Stabilisierungsthese ernüchternd. Lediglich die strukturelle Asymmetrie zwischen CDU und SPD zugunsten der Christdemokraten, die seit 2002 besteht [ASY CDU>SPD seit 2002], dürfte mit hoher Wahrscheinlichkeit weiter Bestand haben. Die Asymmetrie zwischen PDS und SPD, welche seit 2002 besteht [ASY PDS $>$ SPD seit 2002], könnte sich 2016 ändern. So sprechen einige Fakten dafür, dass die Sozialdemokraten seit 1998 erstmals wieder vor den Linken landen. Die Wahrscheinlichkeit für den Fortbestand der Eigenschaftskonstante ist daher maximal moderat. Auch die Konzentration des Kernformats auf CDU, SPD und PDS seit 2006 [COR FOR (CDU/SPD/PDS) seit 2006] ist endlich und könnte mit einem Stimmungsumschwung und einem Aufstieg der AfD oder einer neuen Arithmetik (Rot-RotGrün) verändert werden. Der Fortbestand dieser Eigenschaftskonstante ist daher ebenfalls als moderat einzustufen. Die seit 2006 stabile Segmentierung [SEG seit 2006] könnte sich durch die Veränderungen im Parteiensystem ebenfalls erhöhen. Das Parteiensystem Sachsen-Anhalts ist seit 2006 stabil. Jedoch - und das ist eben die Logik eines Parteiensystems - benötigt es eigentlich nur einer volatilen $\mathrm{Nu}$ ance oder Entscheidung weniger politischer Eliten zur totalen Zersetzung der aktuellen Stabilität. 